\title{
Получение тонких пленок теллурида висмута на полиимидных подложках методом импульсного лазерного осаждения
}

\author{
(C) А.Е. Шупенев, И.С. Коршунов, А.Г. Григорьянц
}

Московский государственный технический университет им. Н.Э. Баумана, 105005 Москва, Россия.

E-mail: ash@bmstu.ru, korshunovivan@gmail.com,mt12@bmstu.ru

Поступила в Редакцию 25 июня 2019 г.

В окончательной редакции 7 ноября 2019 г.

Принята к публикации 7 ноября 2019 г.

В настоящей работе сообщается об особенностях получения тонких термоэлектрических пленок $p-\mathrm{Bi}_{0.5} \mathrm{Sb}_{1.5} \mathrm{Te}_{3}$ и $n-\mathrm{Bi}_{2} \mathrm{Te}_{2.7} \mathrm{Se}_{0.3}$ с характерной толщиной $\sim 300$ нм на полиимидном материале методом импульсного лазерного осаждения. Рассмотрено влияние температуры роста, давления и расстояния между мишенью и подложкой на свойства пленок. Достигнуты высокие значения коэффициента Зеебека 220 и -200 мкВ/К, но факторы электрической мощности для пленок $p$ - и $n$-типа составили 9.7 и 5.0 мкВт/см $\cdot \mathrm{K}^{2}$ соответственно из-за достаточно высоких сопротивлений пленок.

Ключевые слова: тонкие пленки, импульсное лазерное осаждение, термоэлектрический эффект, теллурид висмута, полиимид.

DOI: $10.21883 /$ FTP.2020.03.49038.9196

\section{1. Введение}

Термоэлектрические материалы в виде тонких пленок перспективны для использования в микрогенераторах и измерительной технике. Эффективность термоэлектрических материалов описывается следующим выражением [1]:

$$
Z T=T \cdot \alpha^{2} \sigma / k=P F \cdot T / k,
$$

где $Z T$ - безразмерный параметр термоэлектрической добротности; $\alpha, \rho, k-$ коэффициент Зеебека, удельное электрическое сопротивление и теплопроводность термоэлектрического материала, $T-$ средняя абсолютная температура. С учетом того что определение теплопроводности тонких пленок представляет собой достаточно трудную задачу [2,3], эффективность пленок зачастую описывается электрическим фактором мощности - $P F$. Для генераторных изделий важны высокие значения $P F$, а для измерительной техники важны высокие значения $\alpha$.

Материалы на основе теллурида висмута являются наиболее используемыми и эффективными материалами для комнатного (от 0 до $300^{\circ} \mathrm{C}$ ) диапазона температур, причем наиболее эффективные их виды обладают сложной стехиометрией. Традиционными представителями высокоэффективных материалов на основе теллурида висмута являются $p$ - $\mathrm{Bi}_{0.5} \mathrm{Sb}_{1.5} \mathrm{Te}_{3}$ и $n-\mathrm{Bi}_{2} \mathrm{Te}_{2.7} \mathrm{Se}_{0.3}$ [1]. Серьезную проблему представляет получение таких материалов в виде тонких пленок физическими методами осаждения в вакууме в виду следующих причин.

- Значительная, на много порядков, разница парциальных давлений входящих в формулу элементов приводит к неоднородному разлету испаряемого в условиях вакуума материала мишени;

- значительное реиспарение легколетучих элементов (Te, Se) при высоких - температурах подложки;
— малый коэффициент прилипания Те $(<0.6)$ при температурах подложки менее $300^{\circ} \mathrm{C}$ [4].

Метод импульсного лазерного осаждения (ИЛО) получил свое развитие именно благодаря возможности получения пленок сложной стехиометрии на примере ВТСП соединений $[5,6]$. Высокие энергии частиц разлета $(1-100$ эВ) плазмы нивелируют разницу парциальных давлений [7], что приводит к конгруэнтному переносу материала мишени на подложку. Образованное данным методом плазменное облако значительно отличается своей структурой от большинства методов физического испарения в вакууме и представляет собой положительные ионы осаждаемых частиц, заключенные в единую внешнюю электронную оболочку [8-10]. Таким образом, при достижении плазменного облака осаждаемых частиц сначала происходит активация поверхности за счет электронного облака плазмы, что оказывает положительное влияние на коэффициент прилипания. Возможность использования защитной атмосферы в данном случае используется с целью уменьшения реиспарения легколетучих компонентов пленок [11].

Данный метод успешно применяется для получения тонких пленок теллурида висмута на твердых подложках $[12,13]$. Наилучшие результаты достигаются при температурах подложки в районе $300^{\circ} \mathrm{C}$ и давлении защитных газов в диапазоне $0.1-1.0$ Торр [14-17]. Наблюдается большой разброс по наилучшим достигнутым свойствам термоэлектрических пленок двойных и тройных систем: сообщается о коэффициентах Зеебека от 100 до 220 мкВ/К и электрических факторах мощности от 1 до 35 мкВт/см · К [18-21].

Современной тенденцией является исследование возможностей гибкого исполнения изделий. Поэтому в данной работе исследовался вопрос получения высокоэффективных термоэлектрических тонких пленок 
на гибких полиимидных подложках, которые обладают необходимой термической стойкостью в области температур осаждения, низкой теплопроводностью $(0.14 \mathrm{BT} / \mathrm{M} \cdot \mathrm{K})$, а также коэффициентом термического расширения $\left(12 \cdot 10^{-6} \mathrm{~K}^{-1}\right)$, близким к теллуриду висмута $\left(20 \cdot 10^{-6} \mathrm{~K}^{-1}\right)$.

В связи с этим в настоящей работе изучалась проблема получения высокоэффективных тройных твердых растворов теллурида висмута $p$ - и $n$-типа проводимости в виде тонких пленок на полиимидных подложках методом импульсного лазерного осаждения. Новизной исследования считается получение пленок $p$ - $\mathrm{Bi}_{0.5} \mathrm{Sb}_{1.5} \mathrm{Te}_{3}$ и $n-\mathrm{Bi}_{2} \mathrm{Te}_{2.7} \mathrm{Se}_{0.3}$ на полиимидном материале методом ИЛО, а также рассмотрение влияния расстояния между мишенью и подложкой на термоэлектрические свойства получаемых пленок.

\section{2. Методика эксперимента}

Для осаждения тонких пленок использовался $\mathrm{KrF}$-эксимерный лазер CompexPro102F с длительностью импульса 30 нс и длиной волны 248 нм с углом падения на мишень $45^{\circ}$. Осаждение пленок осуществлялось при различных температурах подложек $20-400^{\circ} \mathrm{C}$, расстояниях от мишени до подложки 70-130 мм, давлениях $10^{-7}-1$ Topp защитного газа (Ar 99.99\%) и плотностях

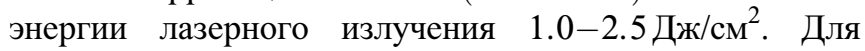
обеспечения однородности слоев использовалось вращение мишени и подложки, а также движение луча по поверхности мишени. Для обеспечения чистоты поверхности пленок производилась полировка поверхности мишеней перед каждым технологическим процессом.

В качестве мишеней использовались цилиндрические монокристаллические слитки $\mathrm{Bi}_{0.5} \mathrm{Sb}_{1.5} \mathrm{Te}_{3}$ и $\mathrm{Bi}_{2} \mathrm{Te}_{2.7} \mathrm{Se}_{0.3}$ диаметром 25 мм, порезанные механически на таблетки высотой $\sim 1$ см. Согласно паспортным данным, материалы $p$ - и $n$-типа обладают коэффициентом Зеебека $205 \pm 5$ и $200 \pm 10 \mathrm{M \kappa B} \cdot \mathrm{K}^{-1}$, электропроводностью $1050 \pm 150$ и $950 \pm 150$ Ом $\cdot \mathrm{cm}^{-1}$ соответственно и теплопроводностью $\sim 1.4 \pm 0.05 \mathrm{BT} \cdot \mathrm{M}^{-1} \cdot \mathrm{K}^{-1}$. В качестве подложек использовался полиимидный материал, аналогичный Kapton, толщиной 100 мкм.

Для определения коэффициента Зеебека использовался стандартный двухзондовый метод, а для определения удельного электрического сопротивления стандартный четырехзондовый метод в линейной геометрии с расстоянием между зондами 1.7 мм. Определение коэффициента Зеебека и удельного электрического сопротивления производилось в одних и тех же участках образцов.

Определение толщины пленок проводилось методами профилометрии в условиях чистой комнаты профилометром KLA-Tencor P17 и атомно-силовой микроскопии с использованием Ntegra Spectra в полуконтактном режиме путем измерения высоты ступеньки между поверхностью пленки и подложки. Элементный анализ проводился для мишеней и наилучших образцов на Quanta SEM с приставкой EDAX.

\section{3. Результаты и обсуждение}

Основная серия экспериментов составила более 100 образцов пленок $p$ - $\mathrm{Bi}_{0.5} \mathrm{Sb}_{1.5} \mathrm{Te}_{3}$ и $n-\mathrm{Bi}_{2} \mathrm{Te}_{2.7} \mathrm{Se}_{0.3}$, полученных на полиимидных подложках. Основные результаты приведены на рисунке. Коэффициент Зеебека пленок напрямую связан со стехиометрией получаемых пленок таким образом, что высоким коэффициентом Зеебека могут обладать только тройные системы, близкие к стехиометричному составу. При низких температурах подложки подвижность атомов на подложке и коэффициент прилипания - низкие [4], что традиционно затрудняет получение стехиометричных многокомпонентных пленок на поверхности при комнатной температуре, и в данном случае обусловливает низкие коэффициенты Зеебека у пленок обоих типов проводимости. Высокие коэффициенты Зеебека наблюдаются уже при достижении температуры в $250^{\circ} \mathrm{C}$ для $\mathrm{Bi}_{0.5} \mathrm{Sb}_{1.5} \mathrm{Te}_{3}$ и $350^{\circ} \mathrm{C}$ для $\mathrm{Bi}_{2} \mathrm{Te}_{2.7} \mathrm{Se}_{0.3}$ и достигают $\sim 200$ мкВ/K. При дальнейшем повышении температуры подложки коэффициент Зеебека начинает снижаться, что обусловлено началом значительных процессов реиспарения легколетучих компонент, и во многих работах более высокие температуры роста не исследуются, хотя характер зависимостей похож [20]. В нашей работе можно наблюдать, что при дальнейшем увеличении температур подложек проявлялась новая тенденция к увеличению коэффициента Зеебека в обоих случаях для образцов $p$ - и $n$-типа. Природа наличия подобных двойных экстремумов коэффициента Зеебека от температуры роста остается предметом дальнейшего изучения. Повышенные температуры роста, $350-400^{\circ} \mathrm{C}$, близки к началу фазовых превращений [22], что позволяет выдвинуть предположение о структурной разнице пленок в наблюдаемых экстремумах, обусловливающей их разные термоэлектрические свойства. Прямое исследование структурных свойств электроннооптическими методами, традиционно применяемыми в случае использования твердых подложек $[15,16]$, затруднено в силу высокой шероховатости и отклонением от плоскости гибких полиимидных подложек. Таким образом, наилучшие образцы пленок $\mathrm{Bi}_{0.5} \mathrm{Sb}_{1.5} \mathrm{Te}_{3}$ были получены при $350^{\circ} \mathrm{C}$ с коэффициентом Зеебека 220 мкВ/K. Следует отметить, что повышенные температуры роста $p$-типа оказались оптимальными и с точки зрения электрического фактора мощности, и, как видно, наилучшие образцы обладают значением $P F$ в $9.7 \mathrm{M \kappa BT} / \mathrm{cm} \cdot \mathrm{K}^{2}$. Это обусловлено падением удельного электрического сопротивления образцов пленок р-типа в несколько раз при температурах $350-400^{\circ} \mathrm{C}$ по сравнению с $250^{\circ} \mathrm{C}$. Для пленок $\mathrm{Bi}_{2} \mathrm{Te}_{2.7} \mathrm{Se}_{0.3}$ рост при повышенных температурах приводит к увеличению удельного электрического сопротивления и не дает преимуществ в итоговом значении $P F$, который составил 5.0 мкВт/см $\cdot \mathrm{K}^{2}$ для пленок, 

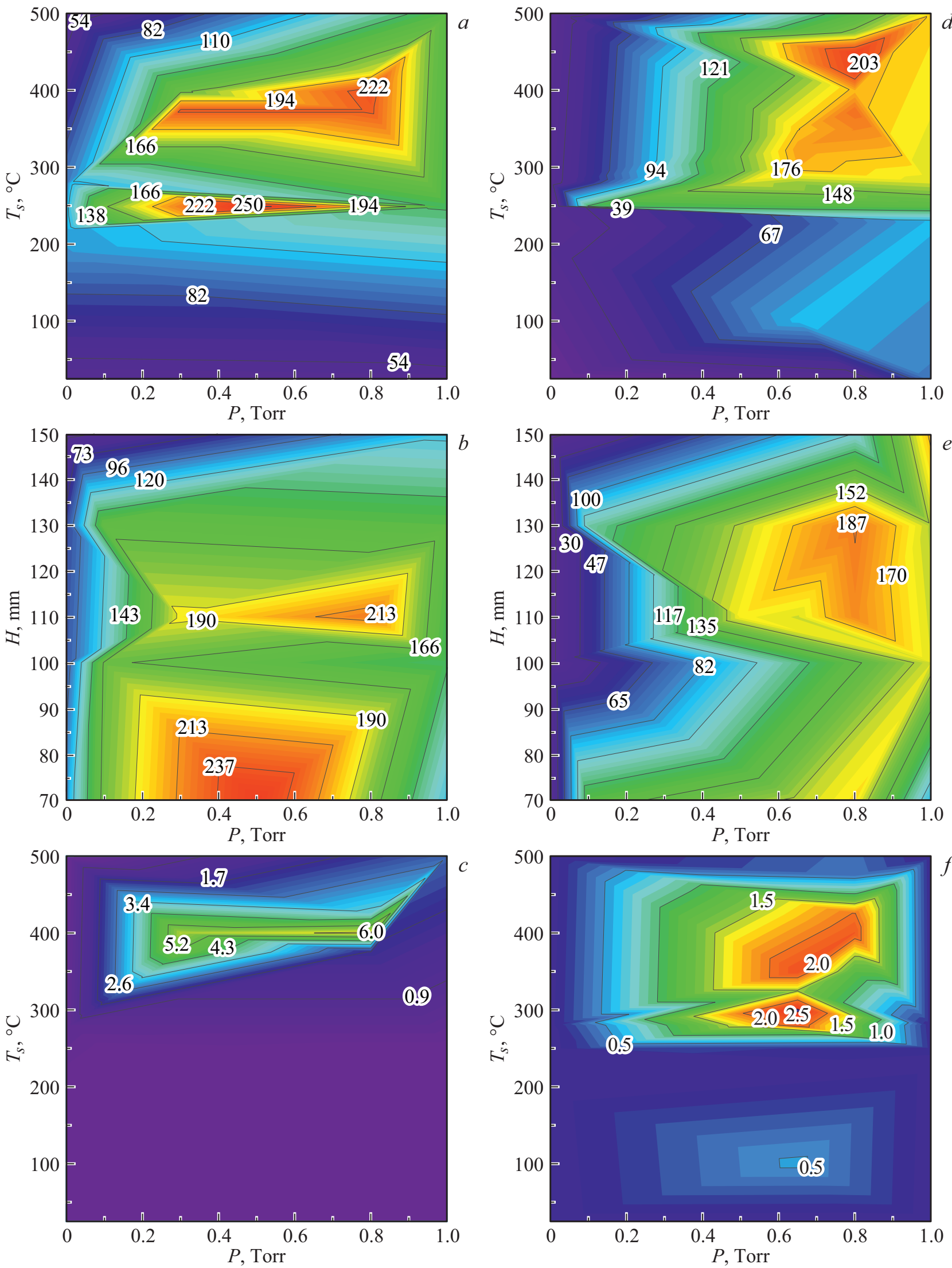

Зависимости коэффициента Зеебека, $\alpha$, от температуры роста, $T_{s}$, и давления защитного газа в камере $P$ для $p$ - $(a)$ и $n$-типов $(d)$; коэффициента Зеебека, $\alpha$, от расстояния между мишенью и подложкой, $H$, и давления защитного газа в камере $P$ для $p$ - $(b)$ и $n$-типов $(e)$; фактора мощности, $P F$, от температуры роста, $T_{s}$, и давления защитного газа в камере $P$ для $p$ - $(c)$ и $n$-типов $(f)$. 
выращенных при $350^{\circ} \mathrm{C}$. Предположительно, большое значение удельного сопротивления, получаемого в пленках, может быть связано с неориентированной структурой полиимидной подложки и снижением подвижности носителей из-за наличия большеугловых границ между кристаллитами.

Остаточное давление в камере контролирует интенсивность реиспарения [11]. Мы проверили экспериментально диапазон давлений от вакуума $10^{-7}$ до 1.0 Торр, и можно видеть, что в условиях вакуума достичь высоких термоэлектрических свойств не получается, как для $p$-типа, так и для $n$-типа. Можно видеть, что область оптимальных давлений для обоих типов находится в диапазоне от 0.2 до 0.8 Торр. Также можно видеть, что пленки $\mathrm{Bi}_{0.5} \mathrm{Sb}_{1.5} \mathrm{Te}_{3}$ значительно менее чувствительны к давлению, чем $\mathrm{Bi}_{2} \mathrm{Te}_{2.7} \mathrm{Se}_{0.3}$, что обусловлено значительно более выраженной летучестью Se и его малым содержанием. В литературе обычно встречается немного более низкий диапазон оптимальных давлений - от 0.1 до 0.6 Торр $[16,20,23]$, чем в данной работе, который составляет 0.4-0.8 Торр. Стоит отметить, что электрическое сопротивление пленок всегда увеличивалось с увеличением давления, и в нашей работе наилучшие образцы обладают высокими значениями удельного электрического сопротивления по сравнению с исходным материалом и лучшими образцами, полученными методом ИЛО в работах [18,19,24], значение $P F$ которых достигает $22-33 \mathrm{м \kappa Вт/см} \cdot \mathrm{K}^{2}$. Следует отметить, что из-за особенностей роста пленок их электрические свойства зависят от морфологии и материала подложки, чем можно объяснить повышенные электрические сопротивления пленок в данной работе и что является предметом дальнейших исследований. Однако принципиальная возможность достижения высоких свойств многокомпонентных пленок теллурида висмута на полиимидных подложках показана в ряде работ $[25,26]$.

Расстояние между подложкой и мишенью влияет на физические процессы разлета газоплазменного облака, а именно интенсивность диффузии легколетучих компонент, энергетическую структуру плазмы, а также геометрические характеристики получаемых пленок [11]. Толщина пленок $h(x, y)$ в случае прямоугольного профиля лазерной обработки в фокальной плоскости описывается следующим выражением [27,28]:

$$
h(x, y)=h_{0}\left(\cos ^{m} \operatorname{arctg}\left(\frac{x}{H}\right)\right)\left(\cos ^{n} \operatorname{arctg}\left(\frac{y}{H}\right)\right),
$$

где $h_{0}-$ максимальная толщина пленки, $n, m-$ коэффициенты разлета плазмы в ортогональных плоскостях, $H$ - расстояние между мишенью и подложкой. Нетрудно увидеть, что увеличение расстояния $H$ способствует получению большей площади однородности толщины пленок, что важно при производстве изделий. Многие лабораторные установки ИЛО не позволяют изменять данное расстояние, которое составляет чаще всего 40-80 мм [12,23,29,30]. Из нашей работы видно, что расстояние $H$ оказывает различное влияние на термоэлектрические свойства осаждаемых пленок в зависимости от давления в камере. Из рисунка видно, что при низких $P$ влияние $H$ не существенно. Примечательно, что расстояние $H$, равное $110 \mathrm{Mм}$, оказалось оптимальным для обоих типов получаемых пленок, что может оказаться удобным в технологии при создании изделий.

\section{4. Заключение}

Экспериментально установлена принципиальная возможность получения качественных тонких пленок $\mathrm{Bi}_{0.5} \mathrm{Sb}_{1.5} \mathrm{Te}_{3}$ и $\mathrm{Bi}_{2} \mathrm{Te}_{2.7} \mathrm{Se}_{0.3}$ методом ИЛО на полиимидных подложках. Достигнуты высокие значения коэффициента Зеебека, 220 и -200 мкВ/K, но факторы электрической мощности для пленок $p$ - и $n$-типа составили 9.7 и $5.0 \mathrm{м \kappa Вт} / \mathrm{cm} \cdot \mathrm{K}^{2}$ соответственно из-за достаточно высоких сопротивлений пленок. Отмечается значительное влияние расстояния между мишенью и подложкой на свойства пленок, а наилучшие результаты получены при значении 110 мм для обоих типов пленок, что вместе с кинематическими особенностями позволило обеспечить высокие термоэлектрические свойства пленок на участке диаметром 80 мм. Повышенные электрические сопротивления и структурные особенности пленок остаются предметом дальнейшего изучения.

\section{Конфликт интересов}

Авторы заявляют, что у них нет конфликта интересов.

\section{Список литературы}

[1] G.J. Snyder, E.S. Toberer. Nature, 7, 105 (2008).

[2] M. Hainan Wang. Int. J. Heat Mass Transfer, 52, 2102 (2009).

[3] A.J. Schmidt, R. Cheaito, M. Chiesa. Rev. Sci. Instrum., 80 (9), 094901 (2009).

[4] P.H. Le, C-N. Liao, C.W. Luo, J. Leu. J. Alloys Compd., 615, $546(2014)$

[5] M.K. Wu, J.R. Ashburn, C.J. Torng, P.H. Hor, R.L. Meng, L. Gao, Z.J. Huang, Y.Q. Wang, C.W. Chu. Phys. Rev. Lett., 58, 908 (1987).

[6] D.H. Lowndes, D.B. Geohegan, A.A. Puretzky, D.P. Norton, C.M. Rouleau. Science, 273, 898 (1996).

[7] R.K. Singh, J. Narayan. Phys. Rev. B, 41 (13), 8843 (1990).

[8] B. Toftmann, J. Schou, S. Canulescu. Appl. Surf. Sci., 278, 273 (2013).

[9] T.N. Hansen, J. Schou, J.G. Lunney. Appl. Phys. A, 69, S601 (1999).

[10] О.А. Новодворский. Автореф. док. дис. (Шатура, ФБГУ ИПЛИТ РАН, 2012).

[11] J. Schou. Appl. Surf. Sci., 255 (10), 5191 (2009).

[12] A. Dauscher, A. Thomy, H. Scherrer. Thin Sol. Films, 280 (1-2), 61 (1996).

[13] A. Dauscher, B. Lenoir, O. Boffoue, A. Jacquot. Proc. SPIE, 4762, 52 (2002). 
[14] A.A. Aziz, M. Elsayed, H.A. Bakr, J. El-Rifai, T. Van der Donck, JP. Celis, V. Leonov, P. Fiorini, S. Sedky. J. Electron. Mater., 39 (9), 1920 (2010).

[15] H.-C. Chang, T.-H. Chen, W.-T. Whanga, C.-H. Chen. J. Mater. Chem. A, 19, 1 (2015).

[16] M. Ohta, H. Obara, A. Yamamoto. Mater. Transactions, 50 (9), 2129 (2009).

[17] Y. Deng, H. Liang, Y. Wang, Z. Zhang, M. Tan, J. Cui. J. Alloys Compd., 509 (18), 5683 (2011).

[18] T.T. Sun, J. Ma, Q.A. Yan, Y. Huang, J. Wang, H.H. Hng. J. Cryst. Growth, 311 (16), 4123 (2009).

[19] E. Symeou, M. Pervolaraki, C.N. Mihailescu, G.I. Athanasopoulos, C. Papageorgiou. Appl. Surf. Sci., 336, 138 (2015).

[20] L.T.C. Tuyen, P.H. Le, C.W. Luo, J. Leu. J. Alloys Compd., 673, 107 (2016).

[21] J. Walachová, R. Zeipl, J. Zelinka, V. Malina. Appl. Phys. Lett., 87 (8), 081902 (2005).

[22] Б.М. Гольцман, В.А. Кудинов, И.А. Смирнов. Полупроводниковые термоэлектрические матриалы на основе $\mathrm{Bi}_{2} \mathrm{Te}_{3}$ (М., Наука, 1972).

[23] P.H. Lea, C.-N. Liao, C.W. Luoc, J-Y. Lind, J. Leua. Appl. Surf. Sci., 285, 657 (2013).

[24] A.Li. Bassi, A. Bailini, C.S. Casari, F. Donati, A. Mantegazza, M. Passoni, V. Russo, C.E. Bottani. J. Appl. Phys., 105, 124307 (2009).

[25] E.I. Rogacheva, A.V. Budnik, M.V. Dobrotvorskaya, A.G. Fedorov. Thin Sol. Films, 612 (1), 128 (2016).

[26] L.M. Goncalves, C. Couto, P. Alpuim, A.G. Rolo. Thin Sol. Films, 518 (10), 2816 (2010).

[27] С.И. Анисимов, Б.С. Лукьянчук. УФН, 172 (3), 301 (2002).

[28] M. Tyunina, K. Sreenivas, C. Bjormander, J. Wittborn, K.V. Rao. Appl. Surf. Sci., 96-98, 831 (1996).

[29] A. Bailini, F. Donati, M. Zamboni, V. Russo. Appl. Surf. Sci., 254 (4), 1249 (2007).

[30] A. Jacquot, B. Lenoir, M.O. Boffoué, A. Dauscher. Appl. Phys. A, 69 (1), S195 (1999).

Редактор А.Н. Смирнов

\section{Pulsed laser deposition of bismuth telluride thin films on polyimide substrates}

\section{A.E. Shupenev, I.S. Korshunov, A.G. Grigoryants}

Bauman Moscow State Technical University, 105005 Moscow, Russia

Abstract Here we report the peculiarities of obtaining $p$ - $\mathrm{Bi}_{0.5} \mathrm{Sb}_{1.5} \mathrm{Te}_{3}$ and $n-\mathrm{Bi}_{2} \mathrm{Te}_{2.7} \mathrm{Se}_{0.3}$ thin thermoelectric films with a thickness of about $300 \mathrm{~nm}$ grown on a polyimide substrate by pulsed laser deposition method. The influence of growth temperature, pressure and target-to-substrate distance on film's thermoelectric properties was investigated. Thermoelectric $p$ - and $n$-type films exhibit high Seebeck coefficient of 220 and $-200 \mu \mathrm{V} / \mathrm{K}$ and low electrical power factors of 9.7 and $5.0 \mu \mathrm{W} / \mathrm{cm} \cdot \mathrm{K}^{2}$ respectively due to relatively high films electric resistances. 